Research Paper

\title{
Investigating Esophageal Stent-Placement Outcomes in Patients with Inoperable Non-Cervical Esophageal Cancer
}

\author{
Mojgan Forootan ${ }^{1}$, Morteza Tabatabaeefar ${ }^{2}$, Nariman Mosaffa ${ }^{3}$, Hormat Rahimzadeh Ashkalak ${ }^{4}$ and \\ Mohammad Darvishis ${ }^{\square}$ \\ 1. Department of Gastroenterology, Gastrointestinal and liver Diseases Research Center (RCGLD), Shahid Beheshti University of Medical Sciences, Tehran, \\ Iran; \\ 2. Department of Radiotherapy, Shahid Beheshti University of Medical Sciences, Tehran, Iran; \\ 3. Department of Immunology, Shahid Beheshti University of Medical Sciences, Tehran, Iran; \\ 4. Department of Internal Medicine, Shahid Beheshti University of Medical Sciences, Tehran, Iran; \\ 5. Infectious Diseases and Tropical Medicine Research Center (IDTMRC), Department of Aerospace and Subaquatic Medicine, AJA University of Medical \\ Sciences, Tehran, Iran.
}

$\square$ Corresponding author: Mohammad Darvishi, Infectious Diseases and Tropical Medicine Research Center (IDTMRC), AJA University of Medical Sciences, Tehran, Iran. Email: mohammaddarvishi@gmail.com Tel: +98 9123060846

(c) Ivyspring International Publisher. This is an open access article distributed under the terms of the Creative Commons Attribution (CC BY-NC) license (https:// creativecommons.org/licenses/by-nc/4.0/). See http://ivyspring.com/terms for full terms and conditions.

Received: 2017.07.10; Accepted: 2017.08.25; Published: 2018.01.01

\begin{abstract}
Background: Esophageal stent insertion in patients with inoperable esophageal cancer is usually accompanied with relatively high adverse symptoms and even mortality. The current study aims at investigating the outcomes of esophageal stenting in patients with inoperable non-cervical esophageal cancer.

Materials and Methods: The current descriptive-analytical research evaluates 25 patients with esophageal cancer. The stent was placed in esophagus based upon endoscopy analysis with or without fluoroscopy and then the stent position and its opening was investigated by making use of CXRand Gastrografin studies. Demographic characteristics, dysphagia scores, stent placement side effects and the dysphagia-related indices were recorded and examined by taking advantage of a questionnaire which was administered pre and post stent insertion.

Results: The study population was comprised of 15 women and 10 men. The individuals' average age was $69.20 \pm 11.25$ years. Dysphagia mean score was lowered from a value of $4.32 \pm 0.80$ to 2.00 \pm 1.22 ( $P$-value $<0.05)$. The prevalent side effects were chest pain (64\%), dysphagia recurrence $(40 \%)$, stent blockage (20\%), stent migration (16\%), Gl bleeding (8\%), and malposition (8\%). Also, it was found out that $64 \%$ of the patients survived for 6 months.

Conclusion: The results indicated that although esophageal stenting is accompanied with side effects in patients with inoperable esophageal cancer, but it is the most frequently used and the most reliable method in relieving dysphagia and improving dysphagia-related life indices.
\end{abstract}

Key words: Non-cervical Esophageal Cancer, Stent Insertion, Dysphagia.

\section{Background}

Esophageal cancer is the third most prevalent malignant disease of the digestive system [1]; it is also the ninth cancer and the sixth forerunning cause of the cancer-related mortalities worldwide [2]. In 2007, 15560 subjects were diagnosed with esophageal cancer in the US out of which 13940 subjects died. The cancer's expression rate differs across the world and it influences a diversity of genders, ages, races and histopathology. According to the studies conducted in this field, the disease is increasing geographically in 
regions from the western to southern Caspian Sea and from the east to northern regions in China. The span of the disease involves sections of Iran, central Asia, Afghanistan, Siberia and Mongolia that is also known as esophageal cancer belt [1]. The outbreak of the esophageal cancer in northern America is indicative of a value equal to 5 to 10 individuals per every 100 thousand persons. This is while the disease outbreak is intensively increasing in Iran and, corresponding to the reports, it shows a value equal to 100 individuals per every 100 thousand individuals [3]. Although the esophageal cancer is more prevalent among the men in western countries but it is found of a higher frequency amongst the women in the northern Iran [4]. Dysphagia is the most important factor behind the esophageal as well as esophagus-stomach juncture cancers which is the major cause of nutritional problems and extremely influences the quality of life in the patients [5]. There are common initial symptoms accompanied with progressive dysphagia including weight loss in a very short period of time. The disease usually becomes incurable when these symptoms show up because the swallowing difficulty usually remains undiagnosed until over $60 \%$ of the esophagus is infiltrated by the cancer. In some of the cases, there are also reported symptoms such as odynophagia (painful dysphagia), chest pain, regurgitation, nausea or pneumonia resulting from aspiration pneumonia and/or tracheoesophageal fistula [1]. Despite the recent advances in the diagnostic methods and treatment techniques, the survival rate of the patients has not undergone so much change during the past four decades $[2,6]$ and the patients with esophageal cancer are usually in a not favorable status in terms of prognosticating signs [7]. The unavailability of general public screening measures has caused the patients to refer to the specialists when their diseases are highly progressed. Thus, about $5 \%$ to $19 \%$ of the patients only live five years post-diagnosis [8]. The entire tumor can be excised only in $45 \%$ of the patients and in a great many of the cases the tumor cells remain at the periphery of the resection margin. As for those patients with esophageal cancer who are incurable and their tumors cannot be removed in surgery, the important complications are dysphagia, malnutrition and tracheoesophageal fistula treatment [1]. Palliative approaches play a considerable role in dysphagia as well as quality of life improvements in patients, but they are not, of course, appropriate candidates for absolute curing of the disease. From the researchers' point of view, the invasive surgical methods inter alia the treatment measures are considered as unfavorable due to their high rate of mortality. Other methods used in treating the dysphagia are malignant stenosis dilatation through guiding wires and blind bougienage, laser therapy, photodynamic treatment, BICAP (Bipolar Circumactive Probe), Ablation, chemical therapy and so forth but these methods require repetition [9]. Finally, the new method applied in improving dysphagia in patients is the insertion of self-expanding metal stents (SEMS) made of Nitinol and stainless steel that are placed by the use of endoscopy or fluoroscopy [10]. Although esophageal stents improve dysphagia, they are usually accompanied by mortality and important side effects such as stent placement side effects, including unconsciousness, perforation, aspiration pneumonia, bleeding, malposition, early side effects emerging one week after stent placement including chest pain, bleeding, tracheal compression, mediastinitis and late side effects expressed after a week of stent placement including stent migration, fistula, GERD (Gastroesophageal Reflux Disease), dysphagia recurrence, blockage due to the tumor growth and bleeding [11, 12]. Since the quality of life and to some extent the survival period in these patients depends on their ability to swallow, thus the improvement in dysphagia plays a significant role in patients' relief and the increase in their quality of life [13]. In studies undertaken in other countries, metallic stents are proposed as confident and easy methods for relieving dysphagia in inoperable patients [14]. Based on the higher prevalence of esophageal cancer in Iran and according to the idea that a study featuring an appropriate study sample volume in this regard is missing from the literature, the researchers decided to design and implement a study aiming at the survey of the outcomes of stent placement in inoperable non-cervical esophageal cancer patients who had been referred to Taleghani and Imam Hussein (peace be upon him) hospitals during the years from 2009 to 2010.

\section{Methods}

\section{Ethics statement and Patients}

The present research is a descriptive-analytical research which was carried out during the years from 2009 to 2010 on patients with esophageal cancer who had referred to Taleghani and Imam Hussein (peace be upon him) Hospitals. The individuals voluntarily participated in the study based on adherence to medical ethics principles and after receiving letters of consent from all of them and informing them well about the study procedures. The study entry scales were as stated in the following words: patients with inoperable non-cervical esophageal disease and dysphagia $\geq 3$, patients with esophageal cancer and weak conditions for undergoing chemo-radiotherapy 
and dysphagia $\geq 3$, high surgery risk patients with dysphagia $\geq 3$, patients with post-surgery tumor recurrence and dysphagia $\geq 3$, dysphagia during or post-chemo-radiotherapy and dysphagia $\geq 3$ and, finally, the existence of fistula. Also, the patients with certain conditions such as degree I and II dysphagia besides the fistula presence and patients with operable esophageal cancer in terms of tumor expansion and the functional status were omitted from the study.

Corresponding to the prior studies and after exerting the entry scales, a total of 25 patients were selected as the study sample volume. The stent placement was carried out by a specialist. The size and the type of the stent was selected subject specifically based on the intensity, the stricture length, the presence or absence of fistula, dysphagia degree as well as according to the patients' conditions and the surgeon's preference. Before inserting the stents, patients' demographic characteristics, disease specifications, stent type, dysphagia degree and the patients' quality of life indices were collected and recorded by means of questionnaires.

Patients' dysphagia was scored by deploying Takita Scoring system (1. the ability to eat normal food, 2. the need to consume liquids with food, 3 . the ability to eat semisolid foodstuff, 4 . the ability to consume only liquids, 5 . the ability to swallow food and 6. complete dysphagia) [15] and dysphagia 3 was considered as being in need of stent insertion. Next, stent placement was performed under conscious sedation and through being guided by endoscope with or without fluoroscopy. In subjects with extreme stenosis, TTS or bougienage was inserted beforehand. Twenty four hours after the abovementioned process, CXR (Chest X-ray) was conducted so as to investigate the stent position and expansion followed by Gastrografin study for the evaluation of possible perforation and in case of normality the subjects were allowed to begin consuming liquids after which they were given semisolid and solid foodstuff. The patients were dismissed in case no new problems appeared and after their dysphagia degree had been scored. In case that the stent was found unopened in CXR tests twenty four hours after placement, the patients were again subjected to endoscopy after one week. Seven days after dismissal and then once every six weeks for six months after the stent insertion date and/or at times when deaths were reported, the patients were visited and checked for dysphagia degree as well as the emergence of new side effects and the gathered information were recorded. Dysphagia-related QOL (quality of life) indices were recorded one day before stenting and then one month post-stent placement by taking advantage of EORTC-QLQ questionnaires.
To investigate the dysphagia improvement, the patients' dysphagia statuses a day before and one week after stent placement were compared. The patients were again subjected to endoscopy in case the stent was found unopened in CXR tests twenty four hours after. During this period of time, the patients could be subjected to chemotherapy, radiotherapy or both based on the preliminary treatment scheme.

\section{Statistical analysis}

The obtained raw data was inserted to SPSS software ver.17 and Kolmogorov-Smirnov test was applied to evaluate the normality of the entire data of concern; in addition appropriate statistical tests were used based upon the normality status of the data. Nonparametric statistical tests such as Wilcoxon and Mann-Whitney U-tests were applied to perform data analyses. Statistical analysis was considered to be statistically significant $\mathrm{P}<0.05$.

\section{Results}

The present research studied 25 patients with esophageal cancer who met the inclusion and exclusion scales. Fifteen subjects $(60 \%)$ were women and 10 subjects $(40 \%)$ were men. The study participants ranged in age from 42 to 86 years of age with an average age of 60.2011 .25 . It was made clear in the examinations that sixteen patients had SCC (squamous cell carcinoma), 8 patients had Adenocarcinoma and one patient had undifferentiated cancer.

Also, the lesion affected region was found in the middle one third of the esophagus in 14 patients (56\%) and also it was found out that the lesion affected 11 patients $(44 \%)$ in the lower one third of the esophagus. Seventy six percent of the patients were found with locally advanced cancers and $24 \%$ were diagnosed with distant metastasis. One patient was also found with fistula in stent placement. In the current study, $28 \%$ of the patients were subjected to pre-stenting radiotherapy which was carried out post-stenting for $24 \%$ of the patients. Also, twenty percent of the patients received chemotherapy pre-stenting which was received pre- and post-stenting by $24 \%$ of the patients. Forty percent of the individuals with esophageal cancer were simultaneously subjected to chemotherapy and radiotherapy. In the current research paper, Ultraflex Stent was used for $48 \%$ of the patients, CHOOSENT was applied for $44 \%$ of the patients and the other types of stents were used for the remaining $8 \%$. Before stent insertion, $24 \%$ of the subjects were subjected to bougienage and $16 \%$ were planted with TTS (Through-The-Scope). The average length of the stents applied in this study was equal to $11 \mathrm{~cm}$ and the 
average diameter of the stents was $8.2 \mathrm{~mm}$. The patients were found to have the following conditions before stenting.

Fifteen patients only had inoperable cancer and dysphagia $\geq 3$ Eight patients underwent stent placement surgeries after chemo-radiotherapy due to the dysphagia recurrence. One patient was subjected to stent placement due to post-surgery dysphagia recurrence and another individual underwent stent insertion as a result of fistula.

The present research paper dealt with the investigation of the dysphagia scores of the patients before and during several weeks post-stent placement. The mean dysphagia score of the patients before stent placement was $4.32 \pm 0.8$. In the present study, the lowest dysphagia mean score, $2 \pm 1.22$, after stent placement belonged to the first week and the highest dysphagia mean score, $2.79 \pm 1.87$, after stent placement belonged to the week eighteen. The mean difference of the dysphagia scores pre- and post-stenting was found to be statistically significant with all the follow-up times after the stent insertion (Table 1).

Table 1. patients' mean dysphagia scores for various weeks preand post-stenting

\begin{tabular}{lll}
\hline Parameter & Dysphagia score & p-value \\
\hline Pre-stenting & $4.32 \pm 0.8$ & - \\
One week after & $2 \pm 1.22$ & 0.001 \\
6 weeks later & $2.35 \pm 1.61$ & 0.001 \\
12 weeks later & $2.62 \pm 1.71$ & 0.002 \\
18 weeks later & $2.79 \pm 1.87$ & 0.007 \\
24weeks after & $2.4 \pm 1.77$ & 0.006 \\
\hline
\end{tabular}

Also, post-stenting patients' dysphagia improvement was also investigated in terms of gender, age, tumor pathology, tumor position, the existence of fistula, staging, chemo-radiotherapy as well as radiotherapy effects, TTS, bougienage and stent type in the current research paper. The results of the examinations are indicative of no significant difference between dysphagia improvement and the evaluated variables (P-value $>0.05)$. The present research paper also assessed the side effects resulting from stent-placement including unconsciousness, perforation, malposition, infiltration to mediastinum, bleeding, aspiration pneumonia, chest pain, tracheal compression, respiratory arrest, mediastinitis, stent migration, fistula creation, GERD, dysphagia recurrence, blockage stemming from tumor growth, feeling of foreign body, food impaction, infection and others. It was made clear in the current study that chest pain is the most common side effect in the patients in such a manner that it was observed in two patients $(8 \%)$ before stenting and in 16 patients $(64 \%)$ after stent placement. Dysphagia recurrence was seen in ten patients $(40 \%)$, blockage as a result of tumor growth was observed in 5 patients $(20 \%)$, stent migration was found in 4 patients (16\%), GERD was seen in 3 patients $(12 \%)$, malposition was observed in 2 patients (8\%), GI bleeding was recorded for 2 patients $(8 \%)$, food impaction was seen in one patient $(4 \%)$ and other side effects were reported for one patient $(4 \%)$.

None of the patients were found with side effects such as unconsciousness, perforation, aspiration pneumonia, mediastinum infiltration, aspiration pneumonia, tracheal compression, respiratory arrest, mediastinitis, fistula formation, feeling of foreign body and infection. Based upon a six-month follow-up analysis, four patients had to undergo stent-placement for a second time. QOL (Quality Of Life) questionnaire was applied pre- and post-stenting to record and compare dysphagia-related indices such as feeding, reflexes, pain, saliva swallowing, feeling of asphyxia, mouth dryness, change in tastes of foods, difficulty in coughing and speaking disorders (Table 2). The results of the performed studies indicated a significant difference between dysphagia indices, feeding, reflex and pain post-stent placement (P-value<0.05); however, no significant difference (P-value $>0.05)$ was found between the other indices (Table 2).

Table 2. indices related to pre- and post-stenting dysphagia in patients under treatment for esophageal cancer

\begin{tabular}{llll}
\hline Dysphagia-related indices & Before stenting & After stenting & P-value \\
\hline Dysphagia & $71.11 \pm 24.00$ & $37.33 \pm 34.97$ & 0.001 \\
Feeding & $42.33 \pm 13.37$ & $22.33 \pm 18.74$ & 0.001 \\
Reflex & $18.00 \pm 22.52$ & $13.33 \pm 21.51$ & 0.030 \\
Pain & $17.33 \pm 21.05$ & $24.88 \pm 25.10$ & 0.030 \\
Saliva swallowing & $17.33 \pm 30.61$ & $8.00 \pm 24.11$ & 0.190 \\
Feeling of asphyxia & $17.33 \pm 29.37$ & $7.99 \pm 14.52$ & 0.084 \\
Mouth dryness & $29.33 \pm 29.37$ & $18.66 \pm 25.60$ & 0.103 \\
Change in taste & $5.33 \pm 12.47$ & $8.00 \pm 24.11$ & 0.280 \\
Difficulty in coughing & $6.66 \pm 19.24$ & $5.33 \pm 15.75$ & 0.650 \\
Talking complications & $1.33 \pm 6.66$ & 0 & 0.310 \\
\hline
\end{tabular}

The average length of survival in 6 month follow-up was 152.4 days with a certainty of $95 \%$ (134.82-169.98). By the end of the research, 16 patients $(64 \%)$ survived while $9(36 \%)$ passed away.

\section{Discussion}

Esophageal stent placement in patients with inoperable esophageal cancer is an appropriate palliative solution which leads to more optimum conditions for this group of the patients. Despite the advantages accompanied with the utilization of such prosthesis for the patients, it is usually followed by a 
lot of side effects and even death. Chest pain is one of the first prevalent side effects after stent insertion and it is often seen in all the patients undergoing esophageal stenting. Although the pain is rather a mild one and it is usually completely relieved within one or two days, but NSAID (Nonsteroidal Anti-Inflammatory Drug) sedatives can also be used [16]. Stent migration and stent blockage are among the other common side effects that are caused by a great many of the reasons. Consumption of cold liquids and lack of complete stent opening are the most important reasons giving rise to stent migration and tumor growth is the major cause of the stent blockage $[17,18]$. In the present study, the researchers dealt with the survey of various kinds of stent placement challenges and side effects in the patients with inoperable non-cervical esophageal cancer and evaluated the patients' post-surgery recovery rate. The patients' post-stenting dysphagia mean scores showed a significant decrease by 2.32 (from $4.33 \pm 0.8$ to $2 \pm 1.22$ ) which is consistent with the results obtained in similar studies undertaken in this field. The most prevalent side effect was chest pain (64\%) which was found to be in a lower rate in comparison to the similar studies performed in Iran (73.7\%) [19]; furthermore, dysphagia indices such as feeding and reflex were found a lot improved in respect to the other studies but the patients' suffered from a higher rate of chest pain which was found to have increased from 17 to 24 . Seventy six percent of the patients had at least a symptom related to stent insertion (plus chest pain) and $56 \%$ had at least one stent-placement symptom (without taking chest pain into account). After chest pain, dysphagia recurrence and stent blockade due to tumor growth were the most frequent, $40 \%$ and $20 \%$ respectively, inter alia the other side effects of the stent placement. Singh et al reported in their study that stent insertion symptoms have been observed in $52 \%$ of the subjects. In their reports, the most important side effects after stent insertion were pain $(38 \%)$, stent blockade $(23 \%)$ and stent migration $(6 \%)$ and as it can be seen the stent placement side effects reported by these researchers are fewer than those which were enumerated in this study [20]. In a retrospective study by Kim et al which was released in 2015, stent placement outcomes were evaluated in 192 patients. In the study carried out by this research group it was shown that mean dysphagia score has reduced by 1.38 (from $3.09 \pm 0.79$ to $1.71 \pm 0.87$ ). In their reports, they pointed out that $22.9 \%$ of these patients had only one symptom. Stent blockade and stent migration, with relative frequencies of $11.9 \%$ and $4.2 \%$, respectively, were the most common side effects. Dysphagia scores calculated by Kim et al have undergone a lower reduction in comparison to what was found herein but the stent utilization symptoms have been fewer in their study and the type of major lesions has also been different [21]. The major side effect in the study conducted by McGaw et al has been stent migration with a relative frequency of $57.1 \%$ which is indicative of a higher rate in respect to our study [22]. In the current research paper, $64 \%$ of the patients survived for a period of six months which has been a little higher in contrast to the study performed by Alizadeh $(63.2 \%)$ [19]. In the study carried out by Hiashia et al which was performed on a sample volume consisted of 66 patients, dysphagia degree reduced from 3.3 to 0.8 ; moreover, $18 \%$ of the patients were found with a second stent blockade resulting from tumor recurrence along with food impaction. Stent blockage for a second time occurred in $23 \%$ of the patients in the present study. Their study results are suggestive of a six-month survival period for $78 \%$ of the patients which is higher than what was observed herein. In a study conducted by Nikhil et al, have reported that 125 patients with esophageal or stomach cancer were investigated for a period of 6 month after stent placement. Dysphagia recurrence was reported in $40 \%$ of the patients which is similar to the current research paper (also 40\%). In some other studies, such as the ones carried out by Freeman and Turkyilmaz $[23,24]$, perforation, aspiration pneumonia and other severe symptoms were reported when stenting but no life threatening side effect was found in the current study. Although before inserting stent-placement needs to be a positron emission tomography (PET) and computed tomography (CT) imaging for response assessment [25-31]. From a general point of view, although there are consistencies between the data reported in various research papers, the statistical parameters regarding the stent placement outcomes, as reported in scientific texts, differ to a great extent and these discrepancies can be attributed to factors such as studies sample volumes, technical factors pertaining to the type of prosthesis and other factors of the like.

\section{Conclusions}

According to the results obtained in the present study and considering the results acquired by the other researchers, it can be concluded that stent insertion can be an appropriate and useful solution to the reduction of dysphagia and improvement of quality of life in patients with inoperable esophageal cancer. However, there is a need for further research featuring larger sample volumes and longer follow-up analyses in order to more clearly elaborate the outcomes derived from stent placement. 


\section{Authors' contributions}

MF and NM participated in the design of the study and statistical analysis, MT and NM participated in the draft of the manuscript, HRA, MT and MF collected cancer specimens, participated in the design of the study, MD as corresponding author conceived the study, and participated in its design, coordination and helped to draft the manuscript. All authors read and approved the final manuscript.

\section{Competing Interests}

The authors have declared that no competing interest exists.

\section{References}

1. Fenton R, Longo D, Fauci A, et al. Harrison's principles of internal medicine. Harrison's Principles of Internal Medicine. 2008.

2. Khushalani NI. Cancer of the esophagus and stomach. Elsevier: Mayo Clinic Proceedings; 2008.

3. Brian G, Denittis A, Christopher GRW. Esophageal cancer. In: halprin EC, Ferez CA, Brady L, editors. Perez and Brady's, Principles and practice of radiation oncology; 5th Edition. Philadelphia: Lippincott Williams and Wilkins; 2008: 51-131.

4. Aledavood A, Anvari K, Sabouri G. Esophageal Cancer in Northeast of Iran. Iranian Journal of Cancer Prevention. 2011;4(3):125-9.

5. Sunde B, Ericson J, Kumagai K, et al. Relief of dysphagia during neoadjuvant treatment for cancer of the esophagus or gastroesophageal junction. Diseases of the esophagus : official journal of the International Society for Diseases of the Esophagus. 2016;29(5):442-7.

6. Siegel R, Naishadham D, Jemal A. Cancer statistics, 2012. CA Cancer J Clin. 2012;62(1):10-29.

7. Tustumi F, Kimura CMS, Takeda FR, et al. Prognostic factors and survival analysis in esophageal carcinoma. Arquivos Brasileiros de Cirurgia Digestiva : ABCD. 2016;29(3):138-41.

8. Berry MF. Esophageal cancer: staging system and guidelines for staging and treatment. Journal of Thoracic Disease. 2014;6(Suppl 3):S289-S97.

9. Mocanu A, Bârla R, Hoara P, et al. Endoscopic palliation of advanced esophageal cancer. Journal of Medicine and Life. 2015;8(2):193-201.

10. Maroju NK, Anbalagan $\mathrm{P}$, Kate $\mathrm{V}$, et al. Improvement in dysphagia and quality of life with self-expanding metallic stents in malignant esophageal strictures. Indian J of Gastroenterology. 2006; 25:262

11. Baron TH. Expandable metal stents for the treatment of cancerous obstruction of the gastrointestinal tract. New England Journal of Medicine. 2001;344(22):1681-7.

12. Verschuur EM, Homs MY, Steyerberg EW, et al. A new esophageal stent design (Niti-S stent) for the prevention of migration: a prospective study in 42 patients. Gastrointestinal endoscopy. 2006;63(1):134-40.

13. Ayres A, Jotz GP, Rieder CR, et al. The Impact of Dysphagia Therapy on Quality of Life in Patients with Parkinson's Disease as Measured by the Swallowing Quality of Life Questionnaire (SWALQOL). International archives of otorhinolaryngology. 2016;20(3):202-6.

14. Shenfine J, McNamee P, Steen N, et al. A pragmatic randomised controlled trial of the cost-effectiveness of palliative therapies for patients with inoperable oesophageal cancer. Health Technol Assess. 2005;9(5):1-121.

15. Besharat $\mathrm{S}$, Jabbari $\mathrm{A}$, Semnani $\mathrm{S}$, et al. Inoperable esophageal cancer and outcome of palliative care. World J Gastroenterol. 2008;14(23):3725-8.

16. Elphick DA, Smith BA, Bagshaw J, et al. Self-expanding metal stents in the palliation of malignant dysphagia: outcome analysis in 100 consecutive patients. Diseases of the esophagus : official journal of the International Society for Diseases of the Esophagus. 2005;18(2):93-5.

17. Keller $\mathrm{R}$, Flieger $\mathrm{D}$, Fischbach $\mathrm{W}$, et al. Self-expanding metal stents for malignant esophagogastric obstruction: experience with a new design covered nitinol stent. Journal of gastrointestinal and liver diseases : JGLD. 2007;16(3):239-43.

18. Sarper A, Oz N, Cihangir C, et al. The efficacy of self-expanding metal stents for palliation of malignant esophageal strictures and fistulas .European journal of cardio-thoracic surgery : official journal of the European Association for Cardio-thoracic Surgery. 2003;23(5):794-8.

19. Mohammad Alizadeh A, Sharifian A, Mirzaee V, et al. Safety and efficacy of stenting in malignant esophageal obstruction. Research in Medicine. 2006;30(3):217-21.
20. Singh $\mathrm{P}$, Singh A, Singh A, et al. Long Term Outcome in Patients with Esophageal Stenting for Cancer Esophagus - Our Experience at a Rural Hospital of Punjab, India .Journal of Clinical and Diagnostic Research : JCDR. 2016;10(12):PC06-PC9.

21. Kim JY, Kim SG, Lim JH, et al. Clinical outcomes of esophageal stents in patients with malignant esophageal obstruction according to palliative additional treatment. Journal of digestive diseases. 2015;16(10):575-84.

22. McGaw C, Alkaddour A, Vega KJ, et al. Stent type used does not impact complication rate or placement time but can decrease treatment cost for benign and malignant esophageal lesions. World Journal of Gastrointestinal Endoscopy. 2016;8(7):338-43.

23. Turkyilmaz A, Eroglu A, Aydin Y, et al. Complications of metallic stent placement in malignant esophageal stricture and their management. Surgical laparoscopy, endoscopy \& percutaneous techniques. 2010;20(1):10-5.

24. Freeman RK, Ascioti AJ, Dake M, et al. An assessment of the optimal time for removal of esophageal stents used in the treatment of an esophageal anastomotic leak or perforation. The Annals of thoracic surgery. 2015;100(2):422-8.

25. Park S, Baratto L,Hatami N. Initial Experience with a New PET/CT System Using SiPM Detectors: Image Quality Comparison with Standard PET/CT. Journal Nuclear Medicine 2017; 57:1331-1331

26. Wu W, Guo WS, Cheng LM, et al. Individual difference of coronal bowing of femur and its influence on the lower limbs alignment after the total knee arthroplasty. Zhonghua Yi Xue Za Zhi. 2017; 97(13):1006-1010.

27. Baratto L, Park SY, Hatami N, et al. 18F-FDG silicon photomultiplier PET/CT: A pilot study comparing semi-quantitative measurements with standard PET/CT. PloS one, 2017;12:e0178936.

28. Sonni I, Minamimoto R, Jamali M, et al. Imaging of tumor-associated system XC- activity with 18F-fluoropropylglutamate (18F-FSPG) PET/CT for intracranial malignancies. Journal Nuclear Medicine 2016; 57: 181-181

29. Park S, Hatami N, Rutledge O. Pilot study of 18F-FSPG vs18F-FDG PET imaging for response assessment in cancer. Journal Nuclear Medicine, 2017; 58:118-118

30. Wu F, Jamali M, Hatami N, et al. 99mTc-MDP scintigraphy vs. $18 \mathrm{~F}-\mathrm{NaF}$ PET/CT for detection of skeletal metastases. Journal Nuclear Medicine 2016; 57. 599-599

31. Sonni I, Park S, Baratto L, et al. Initial Experience with a SiPM-based PET/CT Scanner: Influence of Acquisition Time on Image Quality. Journal of Nuclear Medicine, 2017;58:1369-1369 